\title{
Impfung gegen humane Papillomaviren, die Zervixkarzinome verursachen: Besteht Diskussionsbedarf?
}

\section{Bundesamt für Gesundheit (BAG), Eidgenössische Kommission für Impffragen $(E K I F)^{a}$, Arbeits- gruppe HPV-Impfung ${ }^{b}$}

a Mitglieder: C. Aebi, Bern; R. Anderau, Neuenburg; G. Bachmann, St. Gallen; H. Binz, Solothurn; P. Diebold, Monthey; M. Gallacchi, Melide; U. Heininger, Basel; P. Landry, Neuenburg.

A. Marty-Nussbaumer, Luzern; L. Matter, Basel; K. Mühlemann, Bern; J. Roffler, Genf; C.-A. Siegrist, Genf; S. Stronski Huwiler, Zürich; P. Trefny, Luzern; B. Vaudaux, Lausanne. Sekretariat: Bundesamt für Gesundheit, Sektion Impfungen

b Mitglieder: H. Binz, Solothurn B. Bolliger, St. Gallen; D. Desgrandchamps, Baar; E. Ebnöther, Basel; M. Fehr, Zürich; F. Gurtner, Bern; U. Heininger, Basel; S. Heinzl, Bruderholz; S. Jambresic, Bern; L. Matter, Basel; P.-A. Michaud, Lausanne; D. Nardelli, Lausanne; J. Roffler, Genf; C.-A. Siegrist, Genf;

R. Steffen, Zürich; H. Zimmermann, Bern

\author{
Korrespondenz: \\ Dr. Virginie Masserey Spicher \\ Eidgenössisches Departement \\ des Innern EDI \\ Bundesamt für Gesundheit BAG \\ Sektion Impfungen \\ Schwarztorstrasse 96 \\ CH-3007 Bern \\ Tel. 0313238715 \\ Fax 0313238795
}

virginie.masserey@bag.admin.ch

www.bag.admin.ch
Der erste Impfstoff gegen humane Papillomaviren (HPV), die die meisten Zervixkarzinome verursachen (HPV 16 und 18), wurde in der Schweiz im November 2006 von Swissmedic zugelassen. Im Juni 2007 veröffentlichten das BAG und die EKIF die Empfehlungen für die Impfung gegen das Zervixkarzinom für die Schweiz: Die Impfung wird als Basisimpfung für 11- bis 14-jährige Mädchen sowie während fünf Jahren als Nachholimpfung für Mädchen im Alter von 15 bis 19 Jahren empfohlen. Für junge Frauen ab 20 Jahren wurde zudem eine ergänzende Impfempfehlung abgegeben, wonach die Indikation im Einzelfall zu prüfen ist $[1,2]$. Diese Empfehlungen für die Schweiz wurden gestützt auf eine eingehende Analyse nach vorgegebenen Kriterien beschlossen [3]. In den meisten westlichen Ländern wurden ähnliche Empfehlungen erlassen. Die WHO unterstützt die Aufnahme dieser Impfung in die nationalen Impfprogramme in Europa und hat ein Strategiepapier für die Mitgliedstaaten erarbeitet, um den Prozess zu erleichtern [4]. Mehrere publizierte Studien belegen die Wirksamkeit der Impfung gegen Zervixdysplasien (zervikale intraepitheliale Neoplasien [CIN] Grad 2 und 3), die auf die Impftypen zurückzuführen sind [5-9]. Diese Studien und die Post-Marketing-Überwachung der mehreren Dutzend Millionen Dosen, die seit der Markteinführung vertrieben wurden, haben das gute Sicherheitsprofil dieser Impfstoffe bestätigt $[10,11]$. Trotzdem werden verschiedentlich Stimmen laut, die darauf hinweisen, es seien noch nicht alle Fragen geklärt [12]. Die tatsächlichen Auswirkungen einer neuen Impfung ausserhalb der kontrollierten Bedingungen einer klinischen Studie («effectiveness») können erst nach Jahrzehnten gemessen werden, denn ein Karzinom entwickelt sich erst lange nach einer HPVInfektion. Es ist somit normal, dass bei der Einführung dieses Impfstoffs noch Fragen offen sind. Die EKIF hatte die offenen Fragen wahrgenommen und diskutiert; ihre Überlegungen, die zu den Empfehlungen geführt haben, sind öffentlich zugänglich (www.ekif.ch). Die offenen Fragen stellen jedoch die Angemessenheit der Empfehlungen nicht in Frage. Da die HPV-Impfung der einzelnen Frau und der Gesellschaft Vorteile bringt, ist es gerechtfertigt, diese sichere und wirksame Impfung allen Mädchen zugänglich zu machen, zusammen mit den Informationen, die für eine hinreichende Aufklärung vor dem Entscheid notwendig sind.

Wir möchten im Folgenden nochmals auf diese Fragen eingehen und sie mit Blick auf die aktuellen wissenschaftlichen Erkenntnisse kommentieren.

\section{Krebsprävention}

Der Impfstoff beugt präkanzerösen Läsionen vor, aber wird er langfristig auch Karzinome und Todesfälle verhindern?

Alle Zervixkarzinome entwickeln sich aus einer persistierenden HPV-Infektion der Zervixzellen. Werden die Infektionen und die präkanzerösen Zellveränderungen verhindert, die sich daraus ergeben, werden damit zwangsläufig auch die daraus entstehenden Karzinome verhindert. Dies wird durch den Erfolg der Vorsorgeuntersuchungen formell bestätigt: Die Beseitigung der präkanzerösen Läsionen beugt Karzinomen vor. Natürlich konnte dies durch die bisher verfügbaren Impfstudien nicht belegt werden. Zum einen war die Nachbeobachtungsphase zu kurz, zum anderen wurden die Teilnehmerinnen dieser Studien sehr regelmässig kontrolliert und jede schwere Dysplasie wurde fachgerecht behandelt: Es war somit vorauszusehen, dass weder in der geimpften Gruppe noch in der Placebogruppe ein Karzinom auftreten würde.

\section{Dauer des Impfschutzes}

Wie lange wird der Schutz anhalten?

Es wurde formell nachgewiesen, dass der Impfschutz mindestens fünf Jahre anhält. Mehrere Faktoren weisen jedoch auf eine längere Wirksamkeit hin. Zum einen ist die Impfung bei $>99 \%$ nach vier bis fünf Jahren ohne Anzeichen einer Abschwächung weiterhin wirksam. Zum anderen bleiben die Antikörperspiegel, die diesen Schutz ermöglichen, fünf Jahre nach der Impfung auf einem stabilen Niveau bestehen [13]. Und schliesslich wurde formell nachgewiesen, dass ein immunologisches Gedächtnis induziert wird. Somit würde gegebenenfalls eine Auffrisch- 
dosis genügen, um wieder einen gleich hohen Schutz zu erhalten wie direkt nach der Basisimpfung [14]. Die von den Gesundheitsbehörden verlangten Studien mit einer Nachbeobachtungsphase von mehreren Jahrzehnten werden es ermöglichen, ein allfälliges Nachlassen der Wirksamkeit zu erkennen. Wie bei den Basisimpfungen gegen Tetanus oder Diphtherie müsste dann gegebenenfalls eine Auffrischimpfung empfohlen werden. Im Bereich der Prävention ist es nicht ungewöhnlich, dass Daten erst allmählich verfügbar werden. So wurde zum Beispiel in den 1980er Jahren die Hepatitis-B-Impfung mit der Empfehlung von Auffrischimpfungen eingeführt, die später wieder aufgehoben werden konnten [15]. In der Evaluation der Impfung durch die EKIF wurde die Möglichkeit berücksichtigt, dass bei HPV eine Auffrischimpfung notwendig sein könnte. Dies stellt weder die nachweisliche mehrjährige Wirksamkeit der Impfung noch die Tatsache in Frage, dass schon zahlreiche Frauen aus der Impfung Nutzen ziehen, bevor entschieden wird, ob und in welchen Abständen Auffrischimpfungen notwendig sind.

\section{Natürliche Immunität}

Zahlreiche HPV-Infektionen werden von der natürlichen Immunabwehr erfolgreich bekämpft. Kann die Impfung die natürliche Immunität beeinflussen?

Die natürliche Immunität, die durch eine Infektion induziert wird, ist nicht perfekt, denn eine Frau kann sich wieder mit dem gleichen HPVTyp infizieren, den sie zuvor abgewehrt hat. Hingegen wurde formell nachgewiesen, dass die Impfung gegen HPV 16/18 die Immunität gegen diese Virustypen bei Frauen erhöht, die vor der Impfung bereits einmal infiziert gewesen waren (Boostereffekt) [16]. Die Auswirkungen einer bereits vor der Impfung bestehenden Infektion lassen sich damit allerdings nicht verhindern [17]. Weder die bisher verfügbaren Daten noch die theoretischen Kenntnisse über die Funktionsweise des Immunsystems weisen jedoch darauf hin, dass eine negative Wirkung zu befürchten wäre.

\section{Schutz der Jugendlichen}

Wie kann man die Impfung für 11- bis 14-jährige Mädchen empfehlen, während die Studien, in denen der Schutz vor CIN 2/3 nachgewiesen wurde, mit jungen Frauen im Alter von 16 bis 24 Jahren durchgeführt wurden?

Bei so jungen Mädchen wären Studien mit häufigen gynäkologischen Untersuchungen und einer Nachbeobachtungsphase von fünf bis zehn Jahren nicht angebracht. Die Zulassung der Impfstoffe für die Jugendlichen wurde daher von den Behörden (FDA, EMEA, Swissmedic, WHO) ge- stützt auf den Nachweis gewährt, dass die Impfantikörper, die für die Immunität gegen HPV verantwortlich sind, bei den 9- bis 16-jährigen Mädchen mindestens im gleichen Ausmass induziert werden wie bei den älteren Frauen [18]. Es gibt keinen Grund anzunehmen, dass Antikörperspiegel, die mindestens gleich hoch sind, nicht den gleichen Schutz gewähren, sofern sie bis zum Zeitpunkt der Exposition eine ausreichende Höhe aufweisen. Wie lange die Antikörperspiegel eine ausreichende Höhe beibehalten, wird im Rahmen der Nachkontrolle der klinischen Studien laufend beurteilt. Sollte sich eine Auffrischimpfung als notwendig erweisen, zum Beispiel nach zehn Jahren, könnte diese rechtzeitig empfohlen und den 20- bis 30-jährigen Frauen in der Schweiz problemlos zugänglich gemacht werden.

\section{Vorsorgeuntersuchungen}

Wie wird die Impfung die Compliance bezüglich der Vorsorgeuntersuchungen beeinflussen?

Da die Impfung auf das Risiko von Zervixkarzinomen aufmerksam macht, ist anzunehmen, dass sie eher dazu beitragen wird, für die Wichtigkeit der Vorsorgeuntersuchungen zu sensibilisieren. Im Rahmen der Information über die Impfung wird mit Nachdruck an die Bedeutung der Früherkennung erinnert, da die Impfung nicht vor allen Serotypen schützt, die Krebs verursachen, und regelmässige Vorsorgeuntersuchungen somit weiterhin notwendig sind.

\section{Sexualverhalten}

Wie wird die Impfung das Sexualverhalten der Mädchen beeinflussen?

Auch als 1998 die Hepatitis-B-Impfung für Jugendliche eingeführt wurde, wurde in der Schweiz die Befürchtung geäussert, die Impfung könnte das Sexualverhalten verändern. Die seither durchgeführten Studien haben gezeigt, dass rund 68\% der Jugendlichen in der Schweiz gegen Hepatitis B geimpft sind [19], dass jedoch bezüglich des Alters des ersten Sexualkontakts oder der Zahl der Partner keine Veränderungen zu beobachten sind [20].

\section{Vermehrte Ausbreitung anderer Stämme}

Welchen Einfluss wird die Impfung auf die Häufigkeit der anderen onkogenen Stämme haben?

Es liegen keine Hinweise auf einen Wettbewerb zwischen den verschiedenen Virentypen vor, der dazu führen würde, dass andere Stämme an die Stelle der Impftypen treten. Die durch die Impfung induzierten Antikörper sind für die Impftypen spezifisch, und der Schutz, den sie bieten, erstreckt sich nur auf einige sehr eng verwandte Typen (Typ 31) [21]. Diese Spezifität der Antikör- 
per beschränkt die Wirksamkeit der heutigen HPV-Impfstoffe auf die Prävention von Infektionen mit HPV 16 und 18 (die 70\% der Zervixkarzinome verursachen) sowie mit HPV 31 (das 4\% dieser Karzinome verursacht). In den klinischen Studien wurde nicht beobachtet, dass der Schutz vor den Impftypen die Infektion mit anderen Virustypen signifikant fördert. Im Gegenteil, die durch HPV 16/18 verursachten Läsionen können Infektionen mit anderen HPV-Typen erleichtern (Mischinfektionen), die somit durch die Impfung teilweise verhindert werden könnten [22]. Zudem besetzen die HPV keine spezifische ökologische Nische, da mehrere Viren die gleichen Zellen infizieren können [22]. Schliesslich weist jeder HPV-Typ eine unterschiedliche genetische Neigung auf, persistierende Infektionen zu verursachen und/oder Zellveränderungen auszulösen. Dieses grundsätzliche Verhalten kann durch die Impfung nicht beeinflusst werden. Im Übrigen enthält der Impfstoff kein virales Genmaterial.

\section{Zuwarten?}

Obwohl noch keine Langzeitstudien verfügbar sind, werden bereits breitangelegte Programme lanciert. Sollte nicht besser zugewartet werden?

Die Entwicklung der HPV-Impfstoffe hat über zehn Jahre gedauert, und in Bezug auf das Risiko von Nebenwirkungen besteht ausreichende Erfahrung: Der Impfstoff enthält kein virales Genmaterial und kann somit weder eine Infektion übertragen noch Krebs auslösen. Die Mechanismen der Nebenwirkungen hängen somit mit den Entzündungs- und Immunreaktionen zusammen, die Stunden, Tage oder Wochen nach der Impfung auftreten. Das Interesse an dieser Impfung war so gross, dass sich die Pharmakovigilanzdaten bereits auf mehrere Dutzend Millionen Dosen abstützen können. Obwohl die Überwachung weitergeführt wird, ist es deshalb kaum wahrscheinlich, dass ein Zuwarten während einiger Jahre das hervorragende Verträglichkeitsprofil dieses Impfstoffs verändern wird. Werden die Studienresultate von mehreren Jahrzehnten abgewartet, um die langfristigen Auswirkungen auf das Risiko von präkanzerösen Läsionen und Karzinomen zu überprüfen, würde man den Nutzen der Impfung mehreren Jahrgangsgruppen vorenthalten, obwohl die Wirksamkeit zur Prävention von CIN 2/3 formell nachgewiesen wurde und zu erwarten ist, dass die Impfung auch Karzinome wirksam verhindert.

\section{Kosten-Nutzen-Studien}

Für die Beurteilung des Kosten-Nutzen-Verhältnisses müssen mathematische Modelle für die natürliche Entwicklung der Krankheit entspre- chend den verschiedenen Interventionsstrategien herangezogen werden. Diese Analysen beruhen auf Hypothesen und modellieren den natürlichen Krankheitsverlauf während des Lebens, die Wirksamkeit der Impfung im Verlauf der Zeit, die Wirkung auf die anderen HPV-Stämme oder auf die natürliche Immunität, das Sexualverhalten der Betroffenen und ihrer Partner, die Compliance bezüglich der Vorsorgeuntersuchungen usw. Die gewählten Hypothesen beeinflussen die Ergebnisse dieser Kosten-Nutzen-Studien, die nicht von einem Land auf ein anderes extrapoliert werden können und unter Berücksichtigung der potentiellen Einflüsse interpretiert werden müssen. Im Editorial des NEJM [12] wird an alle Fragen erinnert, die bei der Beurteilung des erwarteten wirtschaftlichen Nutzens berücksichtigt werden müssen. Beruht der Entscheid zur Aufnahme einer Impfung in die nationalen Programme in erster Linie auf wirtschaftlichen Überlegungen, kann es angebracht sein, sich um genauere Antworten auf die offenen Fragen zu bemühen, da diese das Kosten-Nutzen-Verhältnis beeinflussen können.

Das Modell, das die EKIF für ihre Arbeiten benutzt hat, hat sich nachweislich bewährt, um die Epidemiologie des Zervixkarzinoms in der Schweiz genau wiederzugeben (www.ekif.ch). Dieses Modell hat gezeigt, dass das Verhältnis zwischen Kosten und Wirksamkeit trotz der hohen Kosten dieser Impfung (die 2007 auf 236 Franken pro Dosis anstelle der tatsächlichen 140 Franken geschätzt wurden) günstig ist und somit langfristig ein wirtschaftlicher Nutzen zu erwarten ist [23]. In einem Land wie der Schweiz ist zudem die Wirtschaftlichkeit nur ein Argument neben zahlreichen weiteren Faktoren, die bei einem Entscheid für eine Empfehlung berücksichtigt werden. Weitere Faktoren sind zum Beispiel die Krankheitslast, die Wirksamkeit und Sicherheit der Impfung, die Präventions- und Therapiealternativen, der gleiche und gerechte Zugang zu einer Massnahme, die für die Gesundheit des Einzelnen von Nutzen ist usw. Bei all diesen Faktoren hat sich gezeigt, dass die HPV-Impfung einen Nutzen für die Gesundheit der Frauen bringt. Diese Impfung wurde und wird daher von den Mitgliedern der EKIF und von deren Arbeitsgruppe HPV einstimmig unterstützt. Das BAG hat die notwendigen Schritte eingeleitet, um die Dokumente bereitzustellen, die für eine objektive Information erforderlich sind und einen freien Entscheid nach hinreichender Aufklärung ermöglichen. Die EKIF und das BAG überwachen die wissenschaftlichen Daten genau und werden ihre Empfehlungen anpassen, falls sich neue Erkenntnisse ergeben sollten. 
Seit der Publikation der Impfempfehlung im Juni 2007 wurden zusätzliche Resultate verfügbar, die auch einen Schutz vor präkanzerösen Vulvaund Vaginalläsionen [24] sowie vor Läsionen belegen, die durch HPV 31 ausgelöst werden, das in Europa 10\% der präkanzerösen Läsionen und $4 \%$ der Zervixkarzinome verursacht [21]. Andere Studien bestätigen das erhöhte Risiko von Fehlgeburten, Frühgeburten und perinataler Mortalität nach einem Eingriff an der Zervix [25, 26]. Der erwartete Nutzen der HPV-Impfung ist somit noch höher, als Anfang 2007 angenommen wurde.

\section{Literatur}

1 Bundesamt für Gesundheit, Eidgenössische Kommission für Impffragen und Arbeitsgruppe HPV-Impfung. Empfehlungen zur Impfung gegen humane Papillomaviren (HPV). Das Wichtigste in Kürze. Bull BAG. 2007;(25):452-4.

2 Bundesamt für Gesundheit, Eidgenössische Kommission für Impffragen und Arbeitsgruppe HPV-Impfung. Empfehlungen zur Impfung gegen humane Papillomaviren (HPV). Richtlinien und Empfehlungen. Bern: BAG; 2008.

3 Bundesamt für Gesundheit und Eidgenössische Kommission für Impffragen. Impfempfehlungen in der Schweiz: Empfehlungskategorien. Bull BAG. 2005;(45):817-21.

4 WHO Regional Office for Europe. Vaccine-Preventable Diseases and Immunization Programme. Preparing for the Introduction of HPV Vaccine in the WHO European Region. Strategy paper. Geneva: WHO; 2008.

5 Mao C, et al. Efficacy of human papillomavirus-16 vaccine to prevent cervical intraepithelial neoplasia: a randomized controlled trial. Obstet Gynecol. 2006;107(6):1425.

6 Villa LL, Costa RLR, Petta CA, et al. High sustained efficacy of a prophylactic quadrivalent human papillomavirus types 6/11/16/18/ L1 virus-like particle vaccine through 5 years of follow-up. Brit J Cancer. 2006;95:1459-66.

7 The Future II Study Group. Quadrivalent vaccine against human papillomavirus to prevent highgrade cervical lesions. NEJM. 2007;356:1915-27.

8 Paavonen J, Jenkins D, Bosch FX, et al. Efficacy of a prophylactic adjuvanted bivalent L1 virus-like particle vaccine against infection with human papillomavirus types 16 and 18 in young women: an interim analysis of a phase III double-blind randomized controlled trial. Lancet. 2007;369: 2161-70.

9 Ault KA. Effect of prophylactic human papillomavirus L1 virus-like-particle vaccine on risk of cervical intraepithelial neoplasia grade 2, grade 3 , and adenocarcinoma in situ: a combined analysis of four randomised clinical trials. Lancet. 2007; 369:1861-8.

10 www.cdc.gov/vaccinesafety/vaers/FDA_and_CDC_ Information.htm.
11 http://afssaps.sante.fr/htm/10/filcoprs/ cp-gardasil-072008.htm.

12 Haug CJ. Human papillomavirus vaccination reasons for caution. NEJM. 2008;359:861-2.

13 Fraser C, Tomassini JE, Xi L, et al. Modeling the long-term antibody response of a human papillomavirus (HPV) virus-like particle (VLP) type 16 prophylactic vaccine. Vaccine. 2007;25:4324-33. Epub 2007 Mar 12

14 Olsson SE. Induction of immune memory following administration of a prophylactic quadrivalent human papillomavirus (HPV) types 6/11/16/18 L1 virus-like particle (VLP) vaccine. Vaccine. 2007;25:4931-9. Epub 2007 Apr 20.

15 European Consensus Group on Hepatitis B Immunity. Are booster immunisations needed for lifelong hepatitis B immunity? Lancet. 2000;355:561-5.

16 Villa LL. Immunologic responses following administration of a vaccine targeting human papillomavirus Types $6,11,16$, and 18 . Vaccine. 2006;24:5571-83.

17 Hildesheim A. Effect of human papillomavirus 16/18 L1 virus-like particle vaccine among young women with preexisting infection: a randomized trial. JAMA. 2007;298:743-53.

18 Block SL, Nolan T, Sattler C, et al. Comparison of the immunogenicity and reactogenicity of a prophylactic quadrivalent human papillomavirus (types 6, 11, 16, and 18) L1 virus-like particle vaccine in male and female adolescents and young adult women. Pediatrics. 2006;118:2135-45.

19 Bundesamt für Gesundheit. Durchimpfung in der Schweiz 2006. Bull BAG. 2008;(36):619-24.

20 Narring F, Tschumper A, Inderwildi Bonivento L, et al. Santé et styles de vie des adolescents âgés de 16 à 20 ans en Suisse (2002). SMASH 2002: Swiss multicenter adolescent study on health 2002. Raisons de santé 95b. Lausanne: Institut universitaire de médecine sociale et préventive; 2004.

21 Ault KA. Human papillomavirus vaccines and the potential for cross-protection between related HPV types. Gynecol Oncol. 2007;107(2 Suppl 1):S31-3.

22 Méndez F, Muñoz N, Posso H, Molano M, Moreno V, van den Brule AJC. Cervical coinfection with human papillomavirus (HPV) types and possible implications for the prevention of cervical cancer by HPV vaccines. J Infect Dis. 2005;192:1158-65.

23 Szucs TD, Largeron N, Dedes KJ, et al. Cost-effectiveness analysis of adding a quadrivalent HPV vaccine to the cervical cancer screening programme in Switzerland. Curr Med Res Opin. 2008;24:1473-83.

24 Joura EA. Efficacy of a quadrivalent prophylactic human papillomavirus (types 6, 11, 16, and 18) L1 virus-like-particle vaccine against high-grade vulval and vaginal lesions: a combined analysis of three randomised clinical trials. Lancet. 2007;369:1693-702.

25 Albrechtsen S. Pregnancy outcome in women before and after cervical conisation: population based cohort study. BMJ. 2008;337:a1350.

26 Arbyn M. Perinatal mortality and other severe adverse pregnancy outcomes associated with treatment of cervical intraepithelial neoplasia: metaanalysis. BMJ. 2008;337:a1284. 